\title{
Root length determination in primary teeth using Apex Locator and comparing with the Conventional method
}

Neena IE ${ }^{1}$, Ananthraj $A^{2}$, Praveen $\mathrm{P}^{3}$

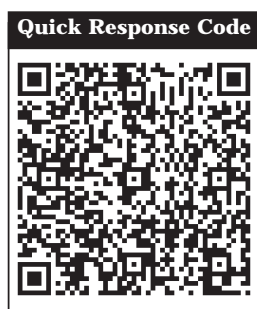

doi: $10.5866 / 2015.7 .10085$

${ }^{1}$ Reader

Department of Pedodontics and Preventive Dentistry College of Dental Sciences,

Davangere-577004, Karnataka, India

2Professor and Head

3Professor

Department of Pedodontics and Preventive Dentistry DAPMRV Dental College,

Bangalore-78, Karnataka, India

\section{Article Info:}

Received: April 11, 2015

Review Completed: May 8, 2015

Accepted: J une 11, 2015

Available Online: April, 2015 (www.nacd.in)

(C) NAD, 2015 - All rights reserved

\section{Email for correspondence:}

neena.ganesh@gmail.com

\begin{abstract}
:
AI M: To comparatively evaluate the accuracy of apex locator and conventional radiographic method in working length determination in primary teeth, in vivo. Working length determination in primary teeth endodontics using apex locator and conventional method for accuracy. METHODOLOGY: This in vivo study was conducted on 30 primary teeth which were indicated for pulpectomy (due to irreversible pulpitis or pulp necrosis) in children aged 5-11 years. in the patients of the age group of 5-11 years. All experimental teeth had adequate remaining tooth structure for rubber dam isolation and radiographically visible canals. In conventional method, standardized intraoral periapical radiographs of teeth the tooth were was taken using paralleling technique. The distance between the source and the tooth, tooth and the films were standardized using X-ray positioning device. The root canal length was also clinically determined with the electronic apex locator RESULT: From the results obtained we can conclude that apex locator methods of determining the root canal length in primary teeth can beconsidered reliablein endodontic treatment of children. CONCLUSION: Apex locator is comparable to conventional radiograph in determining the working length without radiation in the primary teeth. Hence this technique can be safely used as an alternate to conventional radiographic method in determining working length in primary teeth.
\end{abstract}

Key words: Conventional radiography, Apex locator, working length, Primary teeth.

\section{INTRODUCTION}

Pediatric endodontics deals with the management of pulpally involved primary and young permanent teeth in children. Maintaining the integrity and health of the oral tissues is the primary objectives of pulp treatment. It is desirable to attempt to maintain pulp vital ity wherever possible. ${ }^{1}$ In permanent teeth, definitive rules for successful 
endodontic therapy have been established such as compact obturation and apical/coronal seal. In contrast, because of difficulties in estimating the exact root canal length there are no definitive rules for successful endodontic therapy. ${ }^{2}$

Root canal length (working length, root length, tooth length) determination is an important step in root canal therapy. These terms are used interchangeably however they refer to as the distance from the coronal reference point to the point at which canal preparation and obturation should terminate. (Correct working length determination is one of the main factor leading to success in root canal treatment - already mentioned). Endodontic anatomy (root canal morphology) of primary teeth, in particular of molars is difficult to predict because of the resorption, shape, dimension and position of the root apex are often continuously altered. This is the reason that makes it difficult to determine the exact location of the actual apex. To minimize periapical injury and possible damage to the succedaneous tooth, the root length should be carefully determined without exceeding the apex. ${ }^{3}$

Radiographic method described by Ingle is one of the most common and reliable method used in determining the working length, however accuracy is difficult to achieve. In this technique because of apical constriction which cannot be identified and the variables variation in techniques, angulations and exposure distort the image and lead to error. In addition, there is radiation hazard both to the patient and dental personnel. Biased radiographic interpretation may lead to errors. ${ }^{4}$ The purpose of the present study was to compare the working length determined by A pex locator with that of conventional radiographic method for accuracy.

\section{MATERIALS AND METHOD}

This invivo study was conducted on 30 primary teeth which were indicated for pulpectomy in the patients of the age group of 5-11 years, who visited Department of Pediatric and Preventive Dentistry, D.A.P.M.R.V. Dental college. Children wereselected according to the inclusion criteria after obtaining consent from the parents.

All experimental teeth had adequate remaining tooth structure for rubber dam isolation and radio graphically visible canals. A standardized intraoral periapical radiograph of the tooth was taken using conventional method by paralleling technique. The distance between the source and the tooth, tooth and the films were standardized using $X$-ray positioning device.

After administration of local anesthesia, tooth was isolated with rubber dam. Access cavity preparation was done using a round diamond bur under abundant water spray. Pulpal tissue of each tooth was extripated using broach and the root canals were irrigated using sodium hypochlorite solution (percentage concentration). The pulp chamber was dried using sterile cotton.

From the preoperative radiograph, the measurements were done using files with rubber markers which were in sizes ISO 15-20 by keeping $0.5 \mathrm{~mm}$ short of root apex. With this measurements the files were inserted into the canals and conventional radiograph was taken to determine the working length. The root canal length was clinically determined with the el ectronic apex locator. The file was attached to the file holder and the lip clip is attached to patients lip.The file was advanced until the device indicated that constriction has been reached. The root lengths estimated from apex locator were compared with the conventional method of root canal measurements for accuracy. Measurements were subjected to statistical analysis using ANOVA. 


\section{RESULTS}

Table 1: Descriptive statistics of root length $(\mathrm{mm})$ :

\begin{tabular}{llcccc} 
Technique & N & Mean & Std Dev & Min & Max \\
Apex Locator & 90 & 11.79 & 1.70 & 7.00 & 15.50 \\
\hline Conventional Radiograph & 90 & 11.76 & 1.67 & 7.00 & 15.50 \\
\hline
\end{tabular}

Total 90 canals (30 teeth) measurements were taken in this study. Mean of apex locator is 11.79 , conventional radiograph is 11.76 . Table 1 shows the descriptive statistics of root canal lengths $(\mathrm{mm})$ of the total sample. A total of 90 root canals from 30 teeth selected were measured. The mean working length was 11.79 and 11.76 with electronic apex locator and conventional radiograph respectively.

Table 2: Statistical significance between the techniques employed.

ANOVA

Root Length (mm)

\begin{tabular}{lccccc} 
& Sum of Squares & df & Mean Square & F & Sig. \\
Between Groups & 2.585 & 2 & 1.2936 & .453 & .636 \\
\hline Within Groups & 761.367 & 267 & 2.852 & & \\
\hline Total & 763.952 & 269 & & & \\
\hline
\end{tabular}

Table 2 shows the sum of squares between groups as 2.585 , within group as 761.367 , degree of freedom (df) between group as 2 and within group as 267. There was no significant difference in the mean root canal length measurements between the two techniques $(P>0.05)$.

Table 3: Distance between Apex locator and Conventional Radiograph. Proximity Matrix

\begin{tabular}{lcc} 
& \multicolumn{2}{c}{ Matrix File Input } \\
\cline { 2 - 3 } & Apex Locator & Conventional Radiograph \\
Apex Locator & .000 & 6.000 \\
\hline Conventional Radiograph & 6.000 & .0000 \\
\hline
\end{tabular}

In table 3It was observed that the distance between Apex locator and Conventional Radiograph is low.

\section{DISCUSSION}

The establishment of the apical limit of canal preparation is an important phase of root canal treatment. It is generally accepted that canal preparation and filling should be limited within the root canal. ${ }^{6}$ Thus accurate determination of the root canal working length is one of the most important step in endodontic therapy.

Conventional radiographs are needed before, during and immediately after endodontic treatment and then periodically to evaluate the success or failure of the therapy. Therefore repeated exposure to dosage of radiations is required. Numerous animal and human investigations have studied the adverse effects of radiation on those areas in the path of ionizing radiation. These areas include the oral tissue, perioral tissues, head and neck region and human embryos and fetuses in pregnant women. ${ }^{7}$

Conventional radiography as a method of determining the working length has short comings 
in that it depends on the child's co-operation as well as the operator's proficiency. In addition to this, minor degrees of resorption may not be visible, and overlapping by adjacent anatomical structures can obscure the clarity of the image. ${ }^{8}$

The other important problem associated with intra oral periapical radiograph is positioning of the film inside the mouth. Error in placement of film and $\mathrm{x}$-ray cone was eliminated by the design of the positioning apparatus, which allowed the fixation of the x-ray cone and reproducible placements of the experimental films and sensor. ${ }^{9}$ The other problem associated with intra oral periapical radiograph is developing, fixing, drying and storage of the film.

The above problems associated with conventional radiograph were overcome with the introduction of intra oral digital radiograph. A number of fundamental issues need to be resolved before digital radiology can be fully incorporated into clinical practice (Van Der Stelt 1995). The radiological diagnostic techniques used in the present study were chosen to reflect current interest in comparing the performances of digital and conventional radiology and assessing their applicability to root canal measurements. ${ }^{4}$

The first generation apex locators used the resistance method and measured opposition to the flow of direct current. In this pain was felt due to high current in the original machine. Second generation apex locators, also known as I mpedance apex locators, measures opposition to the flow of alternating current or impedance. Major disadvantage is that the canal has to be reasonably free of electro conductive materials to obtain accurate readings. Third generation apex locators are based on Frequency dependence. Disadvantage is sensitive to canal fluid level and needs fully charged battery. Dual frequency alternating current signals are utilized to perform measurements automatically with no calibrations required and can be used in wet and dry environment.

In the present study fifth generation apex locator was used which works on dual frequency type and considered best in any root canal condition. It provides the reader with a digital read out, graphic illustration and an audible signal.

Main advantages of A pex locators are that these measure the root canal length to apical foramen, not to the radiographic apex. Easy and fast to operate, giving good accuracy. Artificial perforations can be recognized and radiation to the patient can be reduced. ${ }^{12}$ It is also emphasized that the use of apex locator alone without the preoperative and postoperative radiograph is not a recommended practice due to the large variation in the tooth morphology and medico legal record keeping requirements. ${ }^{13}$

In the present study 30 primary teeth were taken which would give a statistical significant result and patients selected were in the age group of 5-11 years since they would be having $2 / 3^{\text {rd }}$ of the root length. Files sel ected to measure working length were in the range of $15-20$ similar to the study conducted by Ssu-Kuang Chen et al wherethey have used files of 15-20 size. This is because the tips of the No 10K files were not identifiable because they were less than the $120 \mathrm{mi}$ cron meter required.

A study by Velders et al showed that a size \#15 should be the threshold file size for length measurements and concluded that lengths of size 25 and 20 file in digital images were comparable with the lengths on the conventional film. Another study using density profile plot analysis for digital images noted that file lengths decreased perceptibility with sizes \# 15 and \#10 therefore a size 20 file was chosen for radiographic length determination of true canal length in this study. ${ }^{14}$

Various studies have been carried out where there have been numerous technique employed in working length determination including radiography, apex locator, tactile perception, average tooth length and paper points, but reliability and reproducibility of conventional films have made them gold standard by which all imaging systems are compared. ${ }^{15}$ 
In the present study although there was no significant difference in the mean root length measurements from the three techniques, it was observed that apex locator yields similar measurements to conventional radiograph. In the present study D-Speed film was used for conventional radiographic method. Another study conducted using $D$ and $F$ Speed intraoral radiographs and digital and enhanced digital radiographic images for endodontic file length determination showed no significant difference among the four image types. ${ }^{9}$ Hence the results coincide with our study in use of film speed and accuracy in working length determination. From the results obtained we can conclude that apex locator method of determining the root canal length in primary teeth can be considered reliable and safe in endodontic treatment of children.

\section{CONCLUSION}

The following conclusions were drawn from the present study: Apex locator is comparable to conventional radiograph in determining the working length without radiation in the primary teeth. Hence this technique can be safely used as an alternate to conventional radiographic method in determining working length in primary teeth.

\section{REFERENCES}

1. Tosun G, Erdemir A, Eldeniz AU, Sermet U, Sener $Y$. Accuracy of two electronic apex locators in primary teeth with and without apical resorption: a laboratory study. Int Endo J 2008; 10(11):365-371.

2. Andrej M.Kielbassa, Ursula Muller, Inka Munz and $J$ urgen.Clinical evaluation of the measuring accuracy of ROOT ZX in primary teeth. Oral Surg Oral Med Oral Path Oral Rad Endo 2003; 95(1):94-100.

3. Kumar SS, Chacko Y, and Lakshminarayanan L. A simple model to demonstrate the working of electronic apex locators. Endodontology 2004; 16:50-53.
4. Lozano.A, Forner.L, Liena.C. In vitro comparison of root canal measurements with conventional and digital radiology. Int Endo J 2002; 35:542-550.

5. Edwin T, Parks Gail F, Williamson. Digital Radiography: An Overview. J Contemp Den Prac 2002; 3(4):36-40.

6. Melius B, Jiang J, Zhu Q. Measurement of the Distance Between the Minor Foramen and the Anatomic Apex by Digital and Conventional Radiography. J Endo 2001; 65(10):985-990.

7. Yousef saad A, saad Al-Nazhan. Radiation dose reduction during endodontic therapy: A new technique in combining apex locator and a digital imaging system. J Endo 2000; 26(3):144-147.

8. Harrera Manuela, Abolos Camilo, Planas Amparo J imenz, Llamas Rafael.I nfluence of apical constriction diameter on Root ZX apex locator precision. J Endo 2007; 33(8):995-998.

9. Radel Robert T, Goodell Gary G, McClanahan, Scott B, Cohen Mark E. In Vitro Radiographic Determination of Distance from Working Length Files to Root Ends Comparing Kodak RVG 6000. Schick CDR, and Kodak Insight Film. Basic Research - Technology 2006; 566-568.

10. Gordon M.P.J , Chandler N.P. Electronic apex locators. Int EndoJ 2004; 37:425-437.

11. Saud Al-Nazhan, , Abdulla S. Al Yahya, , Maryam Al-Hindi. Evaluating the accuracy of an electronic root canal measuring device using the clear technique. Saudi Dent J 1993; 5(3):132-135.

12. Federico Lamus, J erald O Katz, Alan G Glaros. Evaluation of a Digital Measurement Tool to Estimate Working Length in Endodontics. J Contem Den Prac 2001; 2(1):1-5.

13. Grimberg F, Banegas G, Chiacchio L, Zmener O. In vivo determination of root canal length: a preliminary report using the Tri Auto ZX apex-locating handpiece. Int Endo J 2002; 35:590-593.

14. Hor D, Krusy S, Attin T. Ex vivo comparison of two electronic apex locators with different scales and frequencies. Int Endo J 2005; 38(12):855-859.

15. Leonardo $M R$, Silva LAB, Nelson-Filho $P$, Silva RAB, Raffaini MSGG.Ex vivo evaluation of the accuracy of two electronic apex locators during root canal length determination in primary teeth. Int EndoJ 2008; 41(4):317321.

\section{Gain quick access to our journal online View our journal at www.nacd.in}

